\title{
Freeness of Hyperplane Arrangements between Boolean Arrangements and Weyl Arrangements of Type $B_{\ell}$
}

\author{
Michele Torielli \\ Department of Mathematics, GI-CoRE GSB \\ Hokkaido University \\ Sapporo, Hokkaido 060-0810, Japan \\ torielli@math.sci.hokudai.ac.jp
}

\author{
Shuhei Tsujie \\ Department of Education \\ Hokkaido University of Education \\ Asahikawa, Hokkaido 070-8621, Japan \\ tsujie.shuhei@a.hokkyodai.ac.jp
}

Submitted: Feb 3, 2020; Accepted: Jul 4, 2020; Published: Jul 24, 2020

(C) The authors. Released under the CC BY-ND license (International 4.0).

\begin{abstract}
Every subarrangement of Weyl arrangements of type $B_{\ell}$ is represented by a signed graph. Edelman and Reiner characterized freeness of subarrangements between type $A_{\ell-1}$ and type $B_{\ell}$ in terms of graphs. Recently, Suyama and the authors characterized freeness for subarrangements containing Boolean arrangements satisfying a certain condition. This article is a sequel to the previous work. Namely, we give a complete characterization for freeness of arrangements between Boolean arrangements and Weyl arrangements of type $B_{\ell}$ in terms of graphs.
\end{abstract}

Mathematics Subject Classifications: 52C35, 32S22, 05C22, 20F55, $13 \mathrm{~N} 15$

\section{Introduction}

A (central) hyperplane arrangement in a vector space is a finite collection of vector subspaces of codimension one. In this article, we are mainly interested in the study of freeness of arrangements (see Section 2 for definitions and basic properties). An arrangement consisting of the reflecting hyperplanes of a Weyl group is called a Weyl arrangement. Saito $[10,11]$ proved that every Weyl arrangement is free. However, no complete characterizations of freeness for Weyl subarrangements are known except for type $A$. Weyl subarrangements of type $A$ are represented by simple graphs and their freeness is characterized in terms of simple graphs (see Section 2). In a similar way, Weyl subarrangements of type $B$ are represented by signed graphs.

In this article, a signed graph is a pair $G=\left(G^{+}, G^{-}\right)$in which $G^{+}=\left(V_{G}, E_{G}^{+}\right)$and $G^{-}=\left(V_{G}, E_{G}^{-}\right)$are simple graphs on a common set of vertices $V_{G}$. Notice that we do not consider graphs with loops, half edges, and so on. See [18] for a general treatment of 
signed graphs. Elements in the set $E_{G}^{+}$(respectively $E_{G}^{-}$) are called positive (respectively negative) edges. Edges are sometimes called links.

Let $\mathbb{K}$ be a field of characteristic zero. For each signed graph $G$ on the vertex set $\{1, \ldots, \ell\}$, we define the signed-graphic arrangement $\mathcal{A}(G)$ in the $\ell$-dimensional vector space $\mathbb{K}^{\ell}$ by

$$
\begin{aligned}
\mathcal{A}(G):=\left\{\left\{x_{i}=0\right\} \mid 1 \leqslant i \leqslant \ell\right\} & \\
& \cup\left\{\left\{x_{i}-x_{j}=0\right\} \mid\{i, j\} \in E_{G}^{+}\right\} \cup\left\{\left\{x_{i}+x_{j}=0\right\} \mid\{i, j\} \in E_{G}^{-}\right\},
\end{aligned}
$$

where $\left(x_{1}, \ldots, x_{\ell}\right)$ denotes a basis of the dual space $\left(\mathbb{K}^{\ell}\right)^{*}$ and, for each linear form $\alpha \in$ $\left(\mathbb{K}^{\ell}\right)^{*},\{\alpha=0\}$ denotes the hyperplane $\operatorname{Ker}(\alpha)$ in $\mathbb{K}^{\ell}$. Note that, in $[2,5,14]$, the authors considered signed graphs with loops and associated to each loop the corresponding coordinate hyperplane. In this article, however, we will always assume that every signedgraphic arrangements contain all the coordinate hyperplanes.

Edelman and Reiner [5, Theorem 4.6] characterized the freeness of Weyl subarrangements between type $A_{\ell-1}$ and $B_{\ell}$ in terms of graphs. Bailey [2] characterized the freeness of signed-graphic arrangements for some cases. Suyama and the authors characterized the freeness of signed-graphic arrangements corresponding to graphs in the case $G^{+} \supseteq G^{-}$as follows.

Theorem 1.1 ([14, Theorem 1.4]). Let $G$ be a signed graph with $G^{+} \supseteq G^{-}$. Then the following conditions are equivalent:

(1) $G$ is balanced chordal.

(2) $\mathcal{A}(G)$ is divisionally free.

(3) $\mathcal{A}(G)$ is free.

Note that condition (2) in Theorem 1.1 is omitted in [14, Theorem 1.4]. However, the verification of freeness was obtained via Abe's division theorem, and therefore condition (2) is also equivalent to the other two conditions.

The main result of this article is a generalization of Theorem 1.1 as follows. Note that a signed-graphic arrangement $\mathcal{A}(G)$ is a Weyl subarrangement of type $B$ containing a Boolean arrangement, and vice versa, where a Boolean arrangement is an arrangement consisting of the coordinate hyperplanes $\left\{x_{i}=0\right\}$.

Theorem 1.2. Let $G$ be a signed graph. Then the following conditions are equivalent:

(1) G satisfies the following three conditions:

(I) $G$ is balanced chordal.

(II) $G$ has no induced subgraphs isomorphic to unbalanced cycles of length three or more.

(III) $G$ has no induced subgraphs which are switching equivalent to the graph in Figure 1. 


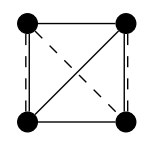

Figure 1: An obstruction to freeness (Dashed line segments denote negative edges)

(2) $\mathcal{A}(G)$ is divisionally free.

(3) $\mathcal{A}(G)$ is free.

The organization of this article is as follows. In Section 2, we review basic properties of freeness of hyperplane arrangements and we recall several results on graphic and signedgraphic arrangements. Moreover, we present necessary conditions for the freeness of a signed-graphic arrangement and we prove the implication $(3) \Rightarrow(1)$ of Theorem 1.2. In Section 3, we introduce a way to construct another signed graph $\tilde{G}$ from a signed graph $G$. The signed graph $\tilde{G}$ satisfies the property $\tilde{G}^{+} \supseteq \tilde{G}^{-}$and hence we may apply Theorem 1.1 to $\tilde{G}$. In Section 4 , we prove Theorem 1.2 in the case $\tilde{G}^{+}$is complete. In Section 5 , we prove a key lemma which states that if $G$ satisfies conditions (I)(II)(III), then $\tilde{G}$ is balanced chordal. This section is an essential part of this article. In Section 6, we prove the implication $(1) \Rightarrow(2)$ of Theorem 1.2 and complete the proof of our main result.

\section{Preliminaries}

\subsection{Hyperplane arrangements}

Let $\mathbb{K}$ be an arbitrary field, $\mathcal{A}$ a central arrangement in the $\ell$-dimensional vector space $\mathbb{K}^{\ell}$. Our main reference on the theory of hyperplane arrangement is [9]. Define the set $L(\mathcal{A})$ by

$$
L(\mathcal{A}):=\left\{\bigcap_{H \in \mathcal{B}} H \mid \mathcal{B} \subseteq \mathcal{A}\right\} .
$$

Note that when $\mathcal{B}$ is empty, the intersection over $\mathcal{B}$ is the ambient vector space $\mathbb{K}^{\ell}$. For subspaces $X, Y \in L(\mathcal{A})$, we define a partial order $\leqslant$ by the reverse inclusion, that is, $X \leqslant Y \stackrel{\text { def }}{\Leftrightarrow} X \supseteq Y$. The set $L(\mathcal{A})$ equipped with the partial order $\leqslant$ forms a geometric lattice. We call $L(\mathcal{A})$ the intersection lattice of $\mathcal{A}$. The rank of $\mathcal{A}$, denoted by $\operatorname{rank}(\mathcal{A})$, is the codimension of the center $\bigcap_{H \in \mathcal{A}} H$. The one-variable Möebius function $\mu: L(\mathcal{A}) \rightarrow \mathbb{Z}$ is defined recursively by

$$
\mu\left(\mathbb{K}^{\ell}\right):=1 \quad \text { and } \quad \mu(X):=-\sum_{Y<X} \mu(Y) .
$$

The characteristic polynomial $\chi(\mathcal{A}, t)$ of $\mathcal{A}$ is defined by

$$
\chi(\mathcal{A}, t):=\sum_{X \in L(\mathcal{A})} \mu(X) t^{\operatorname{dim} X} .
$$


Let $S$ be the ring of polynomial functions on $\mathbb{K}^{\ell}$ and $\operatorname{Der}(S)$ the module of derivations of $S$. Namely,

$\operatorname{Der}(S):=\{\theta: S \rightarrow S \mid \theta$ is $\mathbb{K}$-linear and $\theta(f g)=\theta(f) g+f \theta(g)$ for any $f, g \in S\}$.

Define the module of logarithmic derivations by

$$
D(\mathcal{A}):=\left\{\theta \in \operatorname{Der}(S) \mid \theta\left(\alpha_{H}\right) \in \alpha_{H} S \text { for any } H \in \mathcal{A}\right\},
$$

where $\alpha_{H} \in\left(\mathbb{K}^{\ell}\right)^{*}$ denotes a defining linear form of a hyperplane $H \in \mathcal{A}$. Note that $D(\mathcal{A})$ is a graded $S$-module.

Definition 2.3. An arrangement $\mathcal{A}$ is said to be free if $D(\mathcal{A})$ is a free $S$-module.

For each hyperplane $H \in \mathcal{A}$, we define the restriction $\mathcal{A}^{H}$ by

$$
\mathcal{A}^{H}:=\{H \cap K \mid K \in \mathcal{A} \backslash\{H\}\} .
$$

Note that $\mathcal{A}^{H}$ is an arrangement in $H$.

There are several beautiful results on free arrangements, see for example [15] and [3]. The following results played an important role in [14].

Theorem 2.4 (Abe $\left[1\right.$, Theorem 1.1] (Division Theorem)). Let $H \in \mathcal{A}$. Suppose that $\mathcal{A}^{H}$ is free and $\chi\left(\mathcal{A}^{H}, t\right)$ divides $\chi(\mathcal{A}, t)$. Then $\mathcal{A}$ is free.

Abe's division theorem leads to the notion of divisional freeness.

Definition 2.5. Divisional freeness is defined recursively by the following rules.

(1) The empty arrangements are divisionally free.

(2) If there exists $H \in \mathcal{A}$ such that $\mathcal{A}^{H}$ is divisionally free and $\chi\left(\mathcal{A}^{H}, t\right)$ divides $\chi(\mathcal{A}, t)$, then $\mathcal{A}$ is divisionally free.

Another important notion in the theory of arrangements is supersolvability. We say that $\mathcal{A}$ is supersolvable if the intersection lattice $L(\mathcal{A})$ is supersolvable, that is, there exists a maximal chain of $L(\mathcal{A})$ consisting of modular elements (see [12] for more details). It is well known that every supersolvable arrangement is (divisionally) free (see [8, Theorem $4.2]$ and [1, Theorem 4.4(2)] ).

\subsection{Simple graphs and graphic arrangements}

Let $G=\left(V_{G}, E_{G}\right)$ be a simple graph. Given a subset $W \subseteq V_{G}$, let $G[W]$ denote the induced subgraph on $W$. A chord of a cycle in $G$ is an edge connecting two non-consecutive vertices of the cycle. We say that $G$ is chordal if every cycle of length four or more has a chord. A vertex of $G$ is called simplicial if its neighborhood is a clique, that is, a complete subgraph. An ordering $\left(v_{1}, \ldots, v_{\ell}\right)$ of the vertices of $G$ is called a perfect 
elimination ordering if $v_{i}$ is simplicial in the induced subgraph $G\left[\left\{v_{1}, \ldots, v_{i}\right\}\right]$ for each $i \in\{1, \ldots, \ell\}$.

For a simple graph $G$ on the vertex set $\{1, \ldots, \ell\}$, we define the graphic arrangement $\mathcal{A}(G)$ in $\mathbb{K}^{\ell}$ by

$$
\mathcal{A}(G):=\left\{\left\{x_{i}-x_{j}=0\right\} \mid\{i, j\} \in E_{G}\right\} .
$$

Note that if we identify a simple graph $G$ on $\ell$ vertices with a signed graph $\left(G, \bar{K}_{\ell}\right)$, where $\bar{K}_{\ell}$ denotes the edgeless graph on $\ell$ vertices, then the graphic arrangement $\mathcal{A}(G)$ coincides with the signed-graphic arrangement $\mathcal{A}\left(G, \bar{K}_{\ell}\right)$.

Characterizations of freeness of graphic arrangements are well known as follows.

Theorem 2.6 (Edelman-Reiner [5, Theorem 3.3], Fulkerson-Gross [6, Section 7], Stanley [13, Corollary 4.10]). Let $G$ be a simple graph. Then the following conditions are equivalent:

(1) Gis chordal.

(2) G has a perfect elimination ordering.

(3) $\mathcal{A}(G)$ is supersolvable.

(4) $\mathcal{A}(G)$ is free.

Together with chordal graphs, there is another important family of graphs.

Definition 2.7. Threshold graphs are defined recursively by the following conditions:

(1) The single-vertex graph $K_{1}$ is threshold.

(2) If $G$ is threshold, then the graph obtained by adding an isolated vertex to $G$ is threshold.

(3) If $G$ is threshold, then the graph obtained by adding a dominating vertex to $G$ is threshold, where a vertex is said to be dominating if it is adjacent to the other vertices.

The degree of a vertex $v$ of $G$, denoted $\operatorname{deg}_{G}(v)$, is the number of vertices adjacent to $v$. An ordering $\left(v_{1}, \ldots, v_{\ell}\right)$ of the vertices of $G$ is called a degree ordering if $\operatorname{deg}_{G}\left(v_{1}\right) \geqslant$ $\ldots \geqslant \operatorname{deg}_{G}\left(v_{\ell}\right)$. Note that if $G$ is threshold, then we have either $v_{1}$ is dominating or $v_{\ell}$ is isolated in every degree ordering.

Threshold graphs can also be characterized by forbidden induced subgraphs.

Theorem 2.8 (Golumbic [7, Corollary 5]). A simple graph $G$ is threshold if and only if $G$ is $\left(2 K_{2}, C_{4}, P_{4}\right)$-free, that is, $G$ has no induced subgraphs isomorphic to these three graphs (see Figure 2). 


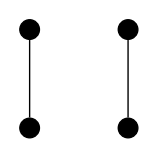

$2 K_{2}$
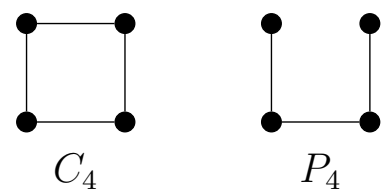

Figure 2: Forbidden induced subgraphs for threshold graphs
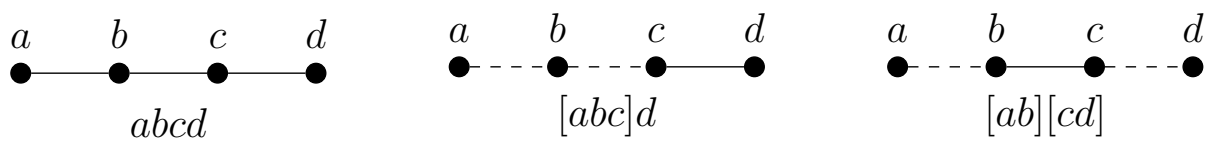

Figure 3: Examples of notation of paths

\subsection{Signed graphs and signed-graphic arrangements}

Given a signed graph $G$ and a subset $W \subseteq V_{G}$, we call $G[W]:=\left(G^{+}[W], G^{-}[W]\right)$ the induced subgraph on $W$. For a vertex $v \in V_{G}$, let $G \backslash v$ denote the induced subgraph on $V_{G} \backslash\{v\}$. Call an edge $e \in E_{G}^{+} \cup E_{G}^{-}$double if $e \in E_{G}^{+} \cap E_{G}^{-}$, otherwise call $e$ single. A path of $G$ is a sequence of distinct vertices $v_{1}, \ldots, v_{k}$ with positive or negative edges $\left\{v_{i}, v_{i+1}\right\} \quad(1 \leqslant i \leqslant k-1)$. For $k \geqslant 3$, a cycle of length $k$ of $G$ is a path $v_{1}, \ldots, v_{k}$ with an additional edge $\left\{v_{1}, v_{k}\right\}$. We use brackets for describing the negative parts of paths and cycles (see Figure 3 for examples).

A chord of a cycle is an edge between two non-consecutive vertices in the cycle. A cycle $C$ is called balanced if $C$ has an even number of negative edges, otherwise $C$ is called unbalanced. A balanced chord of a balanced cycle $C$ is a chord which separates $C$ into two balanced cycles.

Definition 2.9. A signed graph $G$ is said to be balanced chordal if every balanced cycle of length at least four has a balanced chord.

Balanced chordality is a generalization of chordality of simple graphs. Namely, if $G$ is a chordal simple graph on $\ell$ vertices, then the signed graph $\left(G, \bar{K}_{\ell}\right)$ is balanced chordal. We have the following proposition describing the relation of such graphs with freeness.

Proposition 2.10 ([14, Lemma 4.7]). If a signed-graphic arrangement $\mathcal{A}(G)$ is free, then $G$ is balanced chordal.

Proposition 2.11 ([14, Proposition 4.6]). Let $F$ be an induced subgraph of a signed graph $G$. If $\mathcal{A}(G)$ is free, then $\mathcal{A}(F)$ is free.

As for the concept of balanced chordal signed graphs, also the notion of simplicial vertices can be generalized to signed graphs.

Definition 2.12. A vertex $v$ of $G$ is said to be link simplicial if, for any two distinct edges incident to $v$, there exists an edge such that they form a balanced triangle. An ordering $\left(v_{1} \ldots, v_{\ell}\right)$ of the vertices of $G$ is called a link elimination ordering if $v_{i}$ is link simplicial in the induced subgraph $G\left[\left\{v_{1}, \ldots, v_{i}\right\}\right]$ for each $i \in\{1, \ldots, \ell\}$. 
Remark 2.13. Note that a vertex is link simplicial if and only if it is bias simplicial (or signed simplicial) in the signed graph with a loop at every vertex (see [19, 14]).

Zaslavsky completely characterized supersolvability of signed-graphic arrangements. However, we need only the following result in this article.

Theorem 2.14 (Zaslavsky [19, Theorem 2.2]). Suppose that a signed graph G has a link elimination ordering. Then $\mathcal{A}(G)$ is supersolvable.

A switching function $\nu$ of a signed graph $G$ is a function $\nu: V_{G} \rightarrow\{ \pm 1\}$. The signed graph switched by $\nu$ is a signed graph $G^{\nu}$ consisting of the following data:

(1) $V_{G^{\nu}}:=V_{G}$

(2) $E_{G^{\nu}}^{+}:=\left\{\{u, v\} \in E_{G}^{+} \mid \nu(u)=\nu(v)\right\} \cup\left\{\{u, v\} \in E_{G}^{-} \mid \nu(u) \neq \nu(v)\right\}$.

(3) $E_{G^{\nu}}^{-}:=\left\{\{u, v\} \in E_{G}^{+} \mid \nu(u) \neq \nu(v)\right\} \cup\left\{\{u, v\} \in E_{G}^{-} \mid \nu(u)=\nu(v)\right\}$.

We say that $G$ and $G^{\nu}$ are switching equivalent.

Remark 2.15. Note that freeness and the intersection lattice of signed-graphic arrangements is stable under switching since switching acts on arrangements as a coordinate transformation. Moreover, conditions (I) (II) (III) from Theorem 1.2 are also stable under switching.

Let $e$ be an edge of a signed graph $G$ and $H=\left\{x_{i} \pm x_{j}=0\right\}$ the corresponding hyperplane (the sign depends on the sign of $e$ ). Deleting $x_{i}$ in the defining equations of hyperplanes in $\mathcal{A}(G)$ with the relation $x_{i} \pm x_{j}=0$ we obtain the restriction $\mathcal{A}(G)^{H}$. This is again a signed-graphic arrangement, that is, there exists a signed graph $G_{i}$ on $V_{G} \backslash\{i\}$ such that $\mathcal{A}\left(G_{i}\right)=\mathcal{A}(G)^{H}$. If we delete $x_{j}$, then we have another representation of $\mathcal{A}(G)^{H}$ and there exists a signed graph $G_{j}$ on $V_{G} \backslash\{j\}$ such that $\mathcal{A}\left(G_{j}\right)=\mathcal{A}(G)^{H}$. The graphs $G_{i}$ and $G_{j}$ are not isomorphic in general but one can show that they are switching equivalent. Let $G / e$ denote $G_{i}$ or $G_{j}$ and call it the contraction.

Proposition 2.16. Let $G$ be an unbalanced cycle of length three or more. Then $\mathcal{A}(G)$ is non-free.

Proof. We proceed by induction on the length $\ell$ of the cycle $G$. First assume that $\ell=3$. Then the characteristic polynomial is

$$
\chi(\mathcal{A}(G), t)=t^{3}-6 t^{2}+12 t-7=(t-1)\left(t^{2}-5 t+7\right) .
$$

By Terao's factorization theorem [16], we have $\mathcal{A}(G)$ is non-free.

Now suppose that $\ell \geqslant 4$. Assume that $\mathcal{A}(G)$ is free. Fix an edge $e$ and let $H$ be the corresponding hyperplane. Since $G \backslash\{e\}$ has a link elimination ordering, the deletion $\mathcal{A}(G) \backslash\{H\}$ is supersolvable by Theorem 2.14. Using the restriction theorem [9, Corollary 4.47], we have that $\mathcal{A}(G)^{H}$ is free. However, since $G / e$ is an unbalanced cycle of length $\ell-1$, by the induction hypothesis, $\mathcal{A}(G)^{H}$ is non-free, which is a contradiction. Thus $\mathcal{A}(G)$ is non-free. 


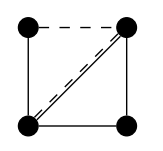

G

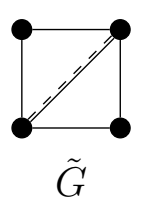

$\tilde{G}$

Figure 4: Example of $G$ and $\tilde{G}$

Proposition 2.17. Let $G$ be the signed graph in Figure 1. Then $\mathcal{A}(G)$ is non-free.

Proof. The characteristic polynomial $\mathcal{A}(G)$ is

$$
\chi(\mathcal{A}(G), t)=t^{4}-12 t^{3}+52 t^{2}-92 t+51=(t-1)(t-3)\left(t^{2}-8 t+17\right) .
$$

By Terao's factorization theorem, we have $\mathcal{A}(G)$ is non-free.

From Proposition 2.10, 2.11, 2.16, and 2.17, we obtain the following lemma, that proves the implication $(3) \Rightarrow(1)$ of Theorem 1.2 ..

Lemma 2.18. Let $G$ be a signed graph. If $\mathcal{A}(G)$ is free, then conditions (I), (II), and (III) hold.

\section{The graph $\tilde{G}$}

In this section, we will describe how to construct a new signed graph $\tilde{G}$ from a given signed graph $G$. Furthermore, we will investigate the properties of $\tilde{G}$. This new graph will play an important role in this article.

Definition 3.19. Let $G=\left(G^{+}, G^{-}\right)$be a signed graph. Define $\tilde{G}:=\left(\tilde{G}^{+}, \tilde{G}^{-}\right)$, where $\tilde{G}^{+}:=G^{+} \cup G^{-}$and $\tilde{G}^{-}:=G^{+} \cap G^{-}$.

Note that $\tilde{G}^{+}$is the underlying simple graph of $G$ and $\tilde{G}^{-}$is the simple graph consisting of the double edges of $G$. Therefore $\tilde{G}$ satisfies $\tilde{G}^{+} \supseteq \tilde{G}^{-}$. Furthermore, we obtain the graph $\tilde{G}$ by replacing the single edges of $G$ with single positive edges. Hence, for any switching function $\nu, G$ and $G^{\nu}$ yield the same graph $\tilde{G}$. However, $G$ and $\tilde{G}$ are not switching equivalent in general (see Figure 4).

Proposition 3.20. If a signed graph $G$ satisfies (I) and (II), then $\tilde{G}^{+}$is chordal.

Proof. Let $C$ be a cycle of $\tilde{G}^{+}$of length at least four. Let $C^{\prime}$ be a cycle of $G$ corresponding to $C$. It suffices to show that $C^{\prime}$ has a chord since every chord of $C^{\prime}$ leads a chord of $C$. If $C^{\prime}$ is unbalanced, then $C^{\prime}$ has a chord or admits a double edge by (II). When the latter holds, we obtain a balanced cycle by changing the sign of the double edge $C^{\prime}$. Hence we may assume that $C^{\prime}$ is balanced. By (I), we have that $C^{\prime}$ has a chord.

Proposition 3.21. Let $G$ be a signed graph. Then a link-simplicial vertex in $G$ is link simplicial in $\tilde{G}$. Moreover, when $G$ has no induced subgraph isomorphic to an unbalanced triangle, a link-simplicial vertex in $\tilde{G}$ is link simplicial in $G$. 
Proof. First we assume that $v$ is link simplicial in $G$ and prove that $v$ is link simplicial in $\tilde{G}$. Take edges $e, e^{\prime}$ of $\tilde{G}$ incident to $v$ such that the other endvertices $u, u^{\prime}$ are distinct.

If $e$ or $e^{\prime}$ is double, then there exists a double edge between $u$ and $u^{\prime}$ since $v$ is a link-simplicial vertex of $G$. Therefore we may choose an edge forming a balanced triangle with $e, e^{\prime}$.

Now we assume that both $e$ and $e^{\prime}$ are positive single edges. Then we have a positive edge in $\tilde{G}$ since $v$ is link simplicial in $G$ and this edge forms a balanced triangle with $e, e^{\prime}$.

Next suppose that $G$ has no induced subgraph isomorphic to an unbalanced triangle and take a link-simplicial vertex $v$ in $\tilde{G}$. We prove that $v$ is link simplicial in $G$. Let $e, e^{\prime}$ be edges of $G$ incident to $v$ such that the other endvertices $u, u^{\prime}$ are different.

If $e$ or $e^{\prime}$ is double, we may prove the assertion in a similar way to the above. Suppose that both $e$ and $e^{\prime}$ are single edges. Since $v$ is link simplicial in $\tilde{G}$, there exists an edge $e^{\prime \prime}$ of $G$ between $u$ and $u^{\prime}$. If $e, e^{\prime}, e^{\prime \prime}$ form an unbalanced triangle, then $e^{\prime \prime}$ is double since $G$ has no induced subgraph isomorphic to an unbalanced triangle. Thus we obtain an edge forming a balanced triangle with $e$ and $e^{\prime}$. Therefore $v$ is link simplicial in $G$.

\section{The case $\tilde{G}^{+}$is complete}

In this section, we consider the case that $\tilde{G}^{+}$is complete.

Proposition 4.22. Suppose that $\tilde{G}^{+}$is complete. If $G$ satisfies (I) and (III), then $\tilde{G}^{-}$is threshold.

Proof. Assume that $\tilde{G}^{-}$is not threshold. Then $\tilde{G}^{-}$has an induced subgraph $H$ isomorphic to $2 K_{2}, C_{4}$, or $P_{4}$ by Theorem 2.8. Let $V_{H}=\{a, b, c, d\}$ be the vertex set of $H$.

First suppose that $H=2 K_{2}$. We may assume that $\{a, b\}$ and $\{c, d\}$ are the edges of $H$. In other words, $\{a, b\}$ and $\{c, d\}$ are the double edges of $G[\{a, b, c, d\}]$. By switching, we may assume that $\{a, d\}$ and $\{b, c\}$ are single positive edges. Considering the balanced cycles $a b c d a$ and $[a b][c d] a$, by condition (I), we may assume that $\{a, c\}$ is a single positive edge and $\{b, d\}$ is a single negative edge, which contradicts condition (III).

Next suppose that $H=C_{4}$ and that $\{a, b\},\{b, c\},\{c, d\},\{d, a\}$ are the double edges of $G[\{a, b, c, d\}]$. By switching, we may assume that the single edges $\{a, c\}$ and $\{b, d\}$ are positive. Consider the balanced cycle $[a b][c d] a$, we have that $\{a, c\}$ or $\{b, d\}$ is negative, which is a contradiction. The case $H=P_{4}$ is similar. Thus the assertion holds.

We are now ready to prove Theorem 1.2 in the case that $\tilde{G}^{+}$is complete.

Proposition 4.23. Suppose that $\tilde{G}^{+}$is complete. Then the following conditions are equivalent:

(1) G has a link elimination ordering.

(2) $\mathcal{A}(G)$ is supersolvable.

(3) $\mathcal{A}(G)$ is free. 
(4) G satisfies (I), (II), and (III).

(5) G satisfies (II) and $\tilde{G}^{-}$is threshold.

Proof. By Theorem 2.14, we have $(1) \Rightarrow(2)$. The implication $(2) \Rightarrow(3)$ is well known, as mentioned before. From Lemma 2.18, the implication $(3) \Rightarrow(4)$ holds. By Proposition 4.22 , we have $(4) \Rightarrow(5)$.

We now show the implication $(5) \Rightarrow(1)$. Let $\left(v_{1}, \ldots, v_{\ell}\right)$ be a degree ordering of $G$. Since $\operatorname{deg}_{G}\left(v_{i}\right)=\operatorname{deg}_{\tilde{G}}\left(v_{i}\right)=\operatorname{deg}_{\tilde{G}^{+}}\left(v_{i}\right)+\operatorname{deg}_{\tilde{G}^{-}}\left(v_{i}\right)$ for every $i \in\{1, \ldots, \ell\}$ and $\tilde{G}^{+}$is complete, the ordering $\left(v_{1}, \ldots, v_{\ell}\right)$ is a degree ordering of $\tilde{G}^{-}$. We proceed by induction on $\ell$. The case $\ell=1$ is trivial. Assume that $\ell \geqslant 2$. It is sufficient to prove that $v_{\ell}$ is a link-simplicial vertex of $G$.

First assume that $v_{\ell}$ is isolated in $\tilde{G}^{-}$. Then $v_{\ell}$ is connected to the other vertices with single edges in $G$. Take two distinct single edges $\left\{v_{i}, v_{\ell}\right\}$ and $\left\{v_{j}, v_{\ell}\right\}$. Since $\tilde{G}^{+}$is complete and the induced subgraph $G\left[\left\{v_{i}, v_{j}, v_{\ell}\right\}\right]$ is not isomorphic to a unbalanced cycle by condition (II), we have that $\left\{v_{i}, v_{j}\right\}$ is a double edge or $G\left[\left\{v_{i}, v_{j}, v_{\ell}\right\}\right]$ is a balanced triangle. In both cases, the vertex $v_{\ell}$ is a link-simplicial vertex of $G$.

Next we suppose that $v_{\ell}$ is not isolated in $\tilde{G}^{-}$. Since $\tilde{G}^{-}$is threshold, $v_{1}$ is a dominating vertex of $\tilde{G}^{-}$. In other words, $v_{1}$ is connected to the other vertices by double edges in $G$. By the induction hypothesis $v_{\ell}$ is link simplicial in $G\left[\left\{v_{2}, \ldots, v_{\ell}\right\}\right]$. Therefore $v_{\ell}$ is link simplicial in $G$.

\section{Balanced chordality of $\tilde{G}$}

This section is devoted to prove the following crucial lemma.

Lemma 5.24. If a signed graph $G$ satisfies conditions (I) (II) (III), then $\tilde{G}$ is balanced chordal.

In order to prove the previous lemma, we need the following proposition.

Proposition 5.25. Suppose that $G$ is a signed graph satisfying conditions (I) (II) (III) and let $C$ be a cycle of $G$ of length four or more admitting two double edges. Let $A, B$ be the vertex sets of the connected components of the graph obtained by removing these double edges from $C$. If $C$ has no chords connecting vertices within $A$ and within $B$, then $C$ has a double chord between a vertex in $A$ and a vertex in $B$.

Proof. We will show the assertion by induction on the length of $C$ and separate the cases. Without loss of generality, we may assume that all the edges of $C$ are positive by switching. We label the vertices of $C$ as shown in Figure 5

Case 1. Assume that $|A|=1$ (Figure 6a). By induction, we will show that every chord $\left\{a_{1}, b_{i}\right\} \quad(2 \leqslant i \leqslant s-1)$ is double.

First assume that the length of $C$ equals four, that is, $s=3$. By condition (I), the balanced cycle $a_{1} b_{1} b_{2} b_{3} a_{1}$ yields a positive chord $\left\{a_{1}, b_{2}\right\}$. Similarly, considering the 


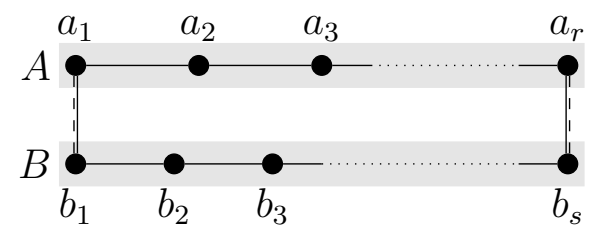

Figure 5: The cycle $C$ with the double edges

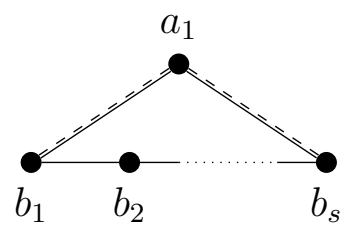

(a)

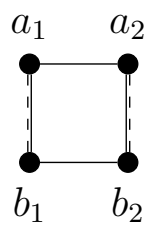

(b)

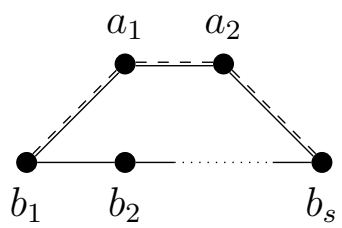

(c)

Figure 6: Cases 1, 2, and 3

balanced cycle $\left[a_{1} b_{1}\right] b_{2}\left[b_{3} a_{1}\right]$, we obtain a negative chord $\left\{a_{1}, b_{2}\right\}$. Thus $\left\{a_{1}, b_{2}\right\}$ is a double chord.

Next, we assume that $s \geqslant 4$. By condition (I), we obtain a positive chord $\left\{a_{1}, b_{i}\right\}$ for some $i \in\{2, \ldots, s-1\}$, which separates our cycle $C$ into two smaller cycles. By the induction hypothesis, we may conclude that the assertion is true.

Case 2. Assume that the length of $C$ equals four. If $|A|=1$ or $|B|=1$, then the assertion holds by Case 1 . Hence we may assume that $|A|=|B|=2$ (Figure 6b). The balanced cycles $a_{1} b_{1} b_{2} a_{2} a_{1}$ and $\left[a_{1} b_{1}\right]\left[b_{2} a_{2}\right] a_{1}$ yield a positive and a negative chord, respectively. If these two chords coincide, then we obtain a desired double chord. If not, the simple graph $\widetilde{G[A \cup B]}^{+}$is complete. Therefore, by Proposition 4.23, we have that the simple graph $G \widetilde{G \cup B]}-$ is threshold. By Theorem 2.8, one of $\left\{a_{1}, b_{2}\right\}$ and $\left\{a_{2}, b_{1}\right\}$ is double.

Case 3. Assume that $|A|=2$ and $\left\{a_{1}, a_{2}\right\}$ is double (Figure 6c). Then the balanced cycle $a_{1} b_{1} \cdots b_{s} a_{2} a_{1}$ yields a positive chord between a vertex in $A$ and a vertex in $B$. By symmetry, we may assume that one of the endvertices is $a_{1}$. Take a positive chord $\left\{a_{1}, b_{m}\right\}$ with $m$ maximal. If $m<s$, then the cycle $a_{1} b_{m} b_{m+1} \cdots b_{s} a_{2} a_{1}$ is of length four or more admitting double edges $\left\{a_{1}, a_{2}\right\}$ and $\left\{a_{2}, b_{2}\right\}$. Applying the result of Case 1 , every $\left\{a_{2}, b_{i}\right\} \quad(m \leqslant i \leqslant s)$ is double. Therefore we may assume that $m=s$, that is, there exists the positive chord $\left\{a_{1}, b_{s}\right\}$. Since the cycle $a_{1} b_{1} \cdots b_{s} a_{1}$ is balanced, every $\left\{a_{1}, b_{i}\right\} \quad(2 \leqslant$ $i \leqslant s)$ is a positive chord. Considering the balanced cycle $\left[a_{1} b_{1}\right] b_{2} \cdots b_{s}\left[a_{2} a_{1}\right]$, we obtain a balanced chord. If one of the endvertices of this chord is $a_{1}$, then this chord is negative and hence we have a double chord. Therefore we may assume that there is a positive chord between $a_{2}$ and a vertex in $B$. Let $\left\{a_{2}, b_{m}\right\}$ be a positive chord with $m$ minimal. By the similar discussion above, we may assume that $m=1$ and we obtain positive chords $\left\{a_{2}, b_{i}\right\} \quad(1 \leqslant i \leqslant s)$. The balanced cycle $\left[a_{1} b_{1}\right] b_{2} \cdots b_{s-1}\left[b_{s} a_{2}\right] a_{1}$ yields a negative chord, which must be double. 


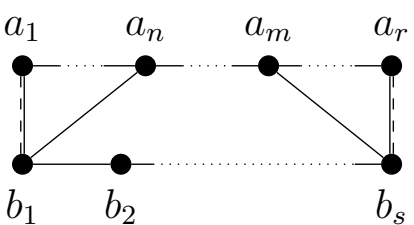

(a)

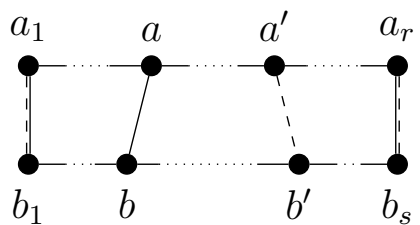

(b)

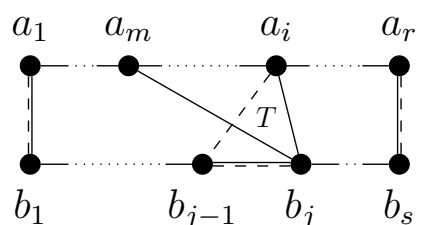

(c)

Figure 7: Case 4

Case 4. Assume that the length of $C$ is at least five. By Case 1, we may suppose that $|A| \geqslant 2$ and $|B| \geqslant 3$. First, we show that there exists a positive chord $\{a, b\}$ such that $a \in A$ and $b \in B \backslash\left\{b_{1}, b_{s}\right\}$. By condition (I), there exists a positive chord between a vertex in $A$ and a vertex in $B$. We may assume that one of the endvertices of this chord is $b_{s}$. Let $\left\{a_{m}, b_{s}\right\}$ be the positive chord with $m$ minimal. Since $s=|B| \geqslant 3$, the balanced cycle $a_{1} b_{1} \cdots b_{s} a_{m} \cdots a_{1}$ is of length four or more and hence has a positive chord. We may assume that one of the endvertices of this chord is $b_{1}$ and take the positive chord $\left\{a_{n}, b_{1}\right\}$ such that $n \leqslant m$ and $n$ is maximal (Figure 7a). The balanced cycle $a_{n} b_{1} \cdots b_{s} a_{m} \cdots a_{n}$ is of length four or more and hence we obtain a desired positive chord $\{a, b\}$. Using the balanced cycle $\left[a_{1} b_{1}\right] b_{2} \cdots b_{s-1}\left[b_{s} a_{r}\right] \cdots a_{1}$, we have a negative chord $\left\{a^{\prime}, b^{\prime}\right\}$ such that $a^{\prime} \in A$ and $b^{\prime} \in B \backslash\left\{b_{1}, b_{s}\right\}$ in a similar way.

If the positive chord $\{a, b\}$ and the negative chord $\left\{a^{\prime}, b^{\prime}\right\}$ coincide, then this is a desired double chord. Otherwise, the positive chord $\{a, b\}$, the negative chord $\left\{a^{\prime}, b^{\prime}\right\}$, the path between $a$ and $a^{\prime}$, and the path between $b$ and $b^{\prime}$ form an unbalanced cycle (Figure 7b). If the length of this unbalanced cycle is four or more, then it has a chord by Proposition 3.20. This chord separates the unbalanced cycle into smaller balanced and unbalanced cycles. Applying the same argument to the smaller unbalanced cycle one after another, we obtain a unbalanced triangle $T$ consisting of a positive and a negative chords of $C$ and an edge $e$ lying in $A$ or $B$. If one of these two chords is double, then this is a desired double chord. Hence we may assume that $e$ is double by condition (II).

If $|A|=2$ and $e=\left\{a_{1}, a_{2}\right\}$, then we have a double chord by Case 3. Hence we may assume that $|A| \geqslant 3$ or the edge $e$ lies in $B$. In both cases, without loss of generality, we may assume that the vertex set of $T$ is $\left\{a_{i}, b_{j-1}, b_{j}\right\}$ for some $i \in\{1, \ldots, r\}$ and $j \in\{3, \ldots, s\}$ by symmetry (Figure $7 \mathrm{c}$ ). Let $m$ be the minimal number such that the chord $\left\{a_{m}, b_{j}\right\}$ exists. By switching, we may assume that this chord is positive. The balanced cycle $a_{1} b_{1} \cdots b_{j} a_{m} \cdots a_{1}$ is of length four or more and satisfies the hypothesis of this proposition. By the induction hypothesis, this cycle has a double chord, which is a desired double chord of $C$.

Proof of Lemma 5.24. Let $C$ be a balanced cycle of $\tilde{G}$ of length at least four. If every edge of $C$ is positive, then $C$ has a positive chord by Proposition 3.20. Hence we may assume that $C$ has at least two negative edges. Let $C^{\prime}$ be a cycle of $G$ corresponding to $C$. Since $C$ has at least two negative edges, the cycle $C^{\prime}$ admits at least two double edges. Hence we may assume that $C^{\prime}$ is a cycle as shown in Figure 5. Without loss of generality, 
we may assume that $|A| \leqslant|B|$.

Let $C^{\prime \prime}$ be a cycle of $G$ on the vertices $a_{1}, b_{1}, a_{r}, b_{s}$, and some vertices in $A$ and $B$ such that there is no chord lying in both $A$ and $B$. Suppose that the length of $C^{\prime \prime}$ is three. This happens only when $|A|=1$ and $C^{\prime \prime}$ is a cycle on the vertices $\left\{a_{1}, b_{1}, b_{s}\right\}$. The edge $\left\{b_{1}, b_{s}\right\}$ leads to a positive chord of $C$. Therefore we may assume that the length of $C^{\prime \prime}$ is at least four. By Proposition 5.25, the cycle $C^{\prime \prime}$ has a double chord between a vertex in $A$ and a vertex in $B$, which is also a double chord of $C^{\prime}$. Choosing a sign of the corresponding double chord of $C$, we may say that $C$ has a balanced chord. Thus $\tilde{G}$ is balanced chordal.

\section{Proof of Theorem 1.2}

The following result played a key role in [14].

Proposition 6.26 ([14, Proposition 6.5 and Lemma 6.9]). Let $G$ be a signed graph with $G^{+} \supseteq G^{-}$. If $G$ is balanced chordal, then one of the following holds:

(i) G has a link-simplicial vertex.

(ii) There exist induced subgraphs $G_{1}$ and $G_{2}$ such that $G_{1} \cup G_{2}=G$ and $G_{1} \cap G_{2}=K_{n}^{ \pm}$, where $K_{n}^{ \pm}:=\left(K_{n}, K_{n}\right)$ denotes a signed graph on $n$ vertices with all possible edges, which is called the complete signed graph.

If $G$ is a signed graph with property (ii) in Proposition 6.26, then the characteristic polynomial of $\mathcal{A}(G)$ is decomposed as

$$
\chi(\mathcal{A}(G), t)=\frac{\chi\left(\mathcal{A}\left(G_{1}\right), t\right) \chi\left(\mathcal{A}\left(G_{2}\right), t\right)}{\chi\left(\mathcal{A}\left(K_{n}^{ \pm}\right), t\right)}
$$

as shown in [14, Theorem 4.10 and Lemma 4.13]. The decomposition is a special case of a property of generalized parallel connections of simple matroids studied by Brylawski [4]. In [17], the second author investigated some arrangements obtained by modular joins (a special kind of generalized parallel connections). For signed graphs, we have the following proposition.

Proposition 6.27 ([17, Theorem 4.11]). Let $\mathfrak{M}$ be the minimal class of signed graphs satisfying the following conditions.

(i) The null graph is a member of $\mathfrak{M}$.

(ii) If $G$ has a link-simplicial vertex $v$ and $G \backslash v \in \mathfrak{M}$, then $G \in \mathfrak{M}$.

(iii) If there exist induced subgraphs $G_{1}$ and $G_{2}$ of a signed graph of $G$ such that $G_{1} \cup G_{2}=$ $G$ and $G_{1} \cap G_{2}=K_{n}^{ \pm}$for some $n$ and $G_{1}, G_{2} \in \mathfrak{M}$, then $G \in \mathfrak{M}$.

Then for every $G \in \mathfrak{M}$, the corresponding arrangement $\mathcal{A}(G)$ is divisionally free. 
Remark 6.28. In this article, we always suppose that $\mathcal{A}(G)$ contains the Boolean arrangements. Therefore the graph is considered to equip all loops in terminology of [17].

Now we are ready to prove the main theorem.

Proof of Theorem 1.2. The implication $(3) \Rightarrow(1)$ is due to Lemma 2.18. The implication $(2) \Rightarrow(3)$ is trivial by the definition of divisional freeness.

We just need to prove the implication $(1) \Rightarrow(2)$. This will be achieved by induction on $\ell$, the number of vertices of $G$. We may assume that $\ell \geqslant 2$ and show that $G \in \mathfrak{M}$, where $\mathfrak{M}$ is defined in Proposition 6.27.

By Lemma 5.24, $\tilde{G}$ is balanced chordal. Therefore $\tilde{G}$ has a link-simplicial vertex $v$ or $\tilde{G}$ is the union of two signed graphs whose intersection is a complete signed graph.

Suppose the former case holds. By Proposition 3.21, the vertex $v$ is link simplicial in $G$. Since the induced subgraph $G \backslash v$ also satisfies conditions (I)(II)(III), the graph $G \backslash v$ belongs to $\mathfrak{M}$ bu the induction hypothesis. Hence $G$ also belongs to $\mathfrak{M}$.

Next we consider the latter case. Then there exist induced subgraphs $G_{1}$ and $G_{2}$ such that $G_{1} \cup G_{2}=G$ and $G_{1} \cap G_{2}=K_{n}^{ \pm}$for some $n$. By the induction hypothesis $G_{1}, G_{2} \in \mathfrak{M}$ and hence $G \in \mathfrak{M}$.

Thus we have proven that $G \in \mathfrak{M}$ by induction. Applying Proposition 6.27, we can conclude that $\mathcal{A}(G)$ is divisionally free.

\section{References}

[1] T. Abe, Divisionally free arrangements of hyperplanes, Inventiones mathematicae 204 (2016), no. 1, 317-346.

[2] G. D. Bailey, Inductively factored signed-graphic arrangements of hyperplanes, Preprint.

[3] A. Bigatti, E. Palezzato, and M. Torielli, New characterizations of freeness for hyperplane arrangements, Journal of Algebraic Combinatorics 51 (2020), no. 2, 297-315.

[4] T. Brylawski, Modular constructions for combinatorial geometries, Transactions of the American Mathematical Society 203 (1975), 1-44.

[5] P. H. Edelman and V. Reiner, Free hyperplane arrangements between $A_{n-1}$ and $B_{n}$, Mathematische Zeitschrift 215 (1994), no. 1, 347-365.

[6] D. Fulkerson and O. Gross, Incidence matrices and interval graphs, Pacific journal of mathematics 15 (1965), no. 3, 835-855.

[7] M. C. Golumbic, Trivially perfect graphs, Discrete Mathematics 24 (1978), no. 1, 105-107.

[8] M. Jambu and H. Terao, Free arrangements of hyperplanes and supersolvable lattices, Advances in Mathematics 52 (1984), no. 3, 248-258.

[9] P. Orlik and H. Terao, Arrangements of Hyperplanes, Grundlehren der mathematischen Wissenschaften, vol. 300, Springer Berlin Heidelberg, Berlin, Heidelberg, 1992. 
[10] K. Saito, On the Uniformization of Complements of Discriminant Loci, RIMS Kôkyûroku 287 (1977), 117 - 137.

[11] K. Saito, Theory of logarithmic differential forms and logarithmic vector fields, Journal of the Faculty of Science, the University of Tokyo. Sect. 1 A, Mathematics 27 (1980), no. 2, 265-291.

[12] R. P. Stanley, Supersolvable lattices, Algebra Universalis 2 (1972), no. 1, 197-217.

[13] R. P. Stanley, An Introduction to Hyperplane Arrangements, Lecture notes, IAS/Park City Mathematics Institute, 2004.

[14] D. Suyama, M. Torielli, and S. Tsujie, Signed graphs and the freeness of the Weyl subarrangements of type $B_{\ell}$, Discrete Mathematics 342 (2019), no. 1, 233-249.

[15] D. Suyama, and S. Tsujie, Vertex-weighted graphs and freeness of $\psi$-graphical arrangements, Discrete and Computational Geometry 61 (2019), 185-197.

[16] H. Terao, Generalized exponents of a free arrangement of hyperplanes and ShepherdTodd-Brieskorn formula, Inventiones mathematicae 63 (1981), no. 1, 159-179.

[17] S. Tsujie, Modular Construction of Free Hyperplane Arrangements, arXiv:1908.01535 (2020).

[18] T. Zaslavsky, Signed graphs, Discrete Applied Mathematics 4 (1982), no. 1, 47-74.

[19] T. Zaslavsky, Supersolvable Frame-matroid and Graphic-lift Lattices, European Journal of Combinatorics 22 (2001), no. 1, 119-133. 